\title{
Determinación del cambio de cobertura agrícola de los municipios de Belén Gualcho, San Marcos de Caiquín y La Virtud, Departamento de Lempira del 2002 al 2014
}

Jessica Gabriela Villatoro Escobar

\section{Resumen}

El objetivo de este estudio fue Determinar los cambios de cobertura agrícola en los Municipios de Belén Gualcho, San Marcos de Caiquin y La Virtud/ Lempira, en un período del 2002-2014. El área de estudio representa un área de 6767 ha.

La metodología de la investigación se realizó con un enfoque cuantitativo siguiendo los siguientes procesos: se reconocieron los principales cultivos existentes en los Municipios abordados, a través del levantamiento de una línea base con el fin de conocer los cultivos pertenecientes a la cobertura agrícola de los Municipios de Belén Gualcho, San Marcos de Caiquin y La Virtud. Se identificó la cobertura agrícola de año 2014 con la ayuda de programas especializados en SIG WEB (Google earth) calculando posteriormente el área agrícola total identificada. Finalmente se utilizó información de cobertura agrícola de los años 2002 y 2014 para calcular el cambio de cobertura agrícola por medio de la fórmula: Tasa de cambio=(área el año actual - área del año anterior)/ (área del año anterior).

De los resultados obtenidos se determinó en el 2002 el área agrícola tomando en cuenta los tres Municipios tenía áreas de $3 \mathrm{Ha}$ hasta $1269 \mathrm{Ha}$. Y en el 2014 áreas de $14.85 \mathrm{Ha}$ hasta $1128.01 \mathrm{Ha}$. La sumatoria total de cobertura agrícola en el año 2002 fue de 2499 Ha y en el 2014 de 10845.58 Ha lo que indica un aumento de la cobertura agrícola con una tasa de cambio de $333.99 \%$. Su valor positivo refleja que en 13 años si se dio un cambio de cobertura agrícola probablemente por el aumento de la población.

Se concluye que al comparar el cambio de cobertura agrícola en un período mayor de 13 años evidencia un cambio en la cobertura agrícola a través de un aumento de 
esta, siendo esta cobertura en mayor proporción cultivos de maíz, frijoles y pasto utilizados en su mayoría para subsistencia y el pasto para alimento de ganado.

Palabras clave: Cobertura agrícola, tasa de cambio, área, línea base, Municipios

Jessica Gabriela Villatoro Escobar (jessicavillatoro_escobar@yahoo.com) Departamento de Ciencia y Tecnologías de la Información Geográfica, Facultad de Ciencias Espaciales, Universidad Nacional Autónoma de Honduras (UNAH). 


\section{Introducción}

La actividad económica principal del Sur de Lempira es la agricultura y la ganadería. En los últimos años se observa el crecimiento agrícola así mismo el mejoramiento de los recursos naturales por las prácticas empleadas; sin embargo hay zonas con fragilidad ambiental, debido a que aún cuentan con malas prácticas agrícolas. Es por esto de gran importancia conocer el uso de la tierra y la evolución del espacio mediante un análisis de inspección y registro de la situación actual, necesarios ambos para explicar la razón de dichos cambios y previo al desarrollo de cualquier propuesta para planificación futura de un determinado espacio. (FAO, 2005).

Al respecto, la Organización de Estados Americanos, al plantear la necesidad de los estudios de usos de la tierra, señala que estos levantamientos son normalmente parte de los componentes de las investigaciones integrales para el desarrollo de los recursos, y supone que estos levantamientos proveen una medida de cómo están siendo utilizados los recursos en relación con su potencial de productividad, tal como es determinado por otros estudios, en particular los realizados sobre suelos y de capacidad productiva de la tierra. (Briceño, 2003).

En diversas investigaciones se han estudiado los cambios en el uso de la tierra comprobando que el uso de datos provenientes de sensores remotos y metodologías de análisis como las cadenas de Markov, permiten detectar problemas asociados con el impacto espacial causado por dichos cambios. Igualmente estas técnicas y métodos facilitan la identificación de las tendencias de los cambios y los resultados obtenidos posibilitan la elaboración de diagnósticos antes o durante el desarrollo de los eventos. (Briceño, 2003).

Además los sensores remotos se ha empleado en estudios de cobertura forestal de muchos países como: México, Colombia, Perú, Paraguay, Bolivia, Brasil y otros. En Perú, en el año 1975 a través de imágenes satelitales (Landsat TM) se reportaron 2 millones de hectáreas entre matorrales y chaparrales, sin embargo en el nuevo mapa forestal elaborado en el año de 1995 se reportan un total de casi 9 millones de bosque. (FAO, 2000). Se plantea como objetivo del presente trabajo la determinación del cambio de cobertura agrícola de los Municipios de Belén Gualcho, San Manuel de Colohete y La Virtud. A diferencia de otros estudios, a continuación se muestran resultados empleando cálculos de áreas y tasas de cambio (terminó económico que muestra variaciones en el tiempo), así mismo los cultivos más predominantes de la región. Dichos resultados constituyen información 
pertinente para conocer si se han dado cambios entorno a la cobertura agrícola en un período de tiempo del 2002 al 2014.

\section{Metodología}

La Zona de estudio conformada por los Municipios: Belén Gualcho, San Marcos de Caiquin y La Virtud, se encuentra ubicada en la Subcuenca del Río Mocal al Sur del Departamento de Lempira.

Mediante una serie de fases a seguir, se determinó el cambio de la cobertura agrícola de 3 Municipios: Belén Gualcho, San Marcos de Caiquin y La Virtud, registrando aspectos del fenómeno de interés de tal manera que esos registros fueran cuantificados. Así mismo la observación e identificación agrícola fue importante, acompañada de la recolección y cruces de datos. Por tanto se logró realizar un sondeo sobre tipos de cultivos, así como interpretar visualmente la agricultura además de generar y analizar datos obtenidos de la fórmula que determina la tasa de cambio y que se muestra a continuación:

$$
\text { Cambio }=\frac{\text { área del año actual }- \text { área del año anterior x } 100}{\text { área del año anterior }}
$$

\section{A. Población y muestras}

El área total del estudio es de 6,767.00 ha. La cual se obtuvo a través del programa especializado de sistemas de información geográfica: ArcGis, que permite calcular el área geográfica de un lugar, en este caso el área total incluye tres Municipios: Belén Gualcho, San Marcos de Caiquin y La Virtud. Además se generó una línea base de los tipos de cultivo aplicando una encuesta obtenida por medio del total de la población rural del área de estudio y aplicando la fórmula estadística $n=N^{*} S^{\wedge} 2 /\left[(N-1)^{*} B^{\wedge} 2 / z^{\wedge} 2+S^{\wedge} 2\right]$ donde:

$\mathrm{N}=$ tamaño de la población,

n=tamaño de la muestra,

$S=$ varianza,

$B=$ tamaño del error,

$z=$ nivel de confianza, en este caso se utilizó un nivel de confianza del $90 \%$. 


\section{B. Fases desarrolladas}

- Verificación en campo

- Se identificaron los principales cultivos existentes en los Municipios abordados, a través de una línea base con el fin de conocer los cultivos pertenecientes a la cobertura agrícola de los Municipios de Belén Gualcho, San Marcos de Caiquin y La Virtud, Lempira.

- Identificación de cobertura agrícola

- Con la ayuda del programa SIG (google earth) se identificaron zonas agrícolas de los Municipios de Belén Gualcho, San Marcos de Caiquin y La Virtud, Lempira en un período del 2002-2014.Posteriormente se realizaron cálculos de áreas de las zonas agrícolas identificadas.

- Estimación de cambio de cobertura agrícola

- Se calculó el área de las zonas agrícolas ya identificadas para los años 2002-2014 en Belén Gualcho, San Marcos de Caiquin y La Virtud, utilizando para identificar la cobertura agrícola del año 2014, el Google earth e información en modelo vector, así mismo para el año 2002 información en modelo vector, visualizada con SIG escritorio (ArcGis).

- Posteriormente se obtuvo cambio de cobertura agrícola empleando la tasa de cambio para períodos del 2002-2014 con la fórmula:

$$
\text { Cambio }=\frac{\text { área del año actual }- \text { área del año anterior x } 100}{\text { área del año anterior }}
$$

- $\quad$ Análisis de Resultados

- Los resultados fueron tratados a través de programas especializados en Sistemas de Información Geográfica y SIG cliente web (google earth), utilizando una escala grande para tener mayor detalle y la cual se puede observar en los mapas elaborados. Dichos mapas fueron interpretados para su representación de cartografía digital y finalmente para un análisis.

\section{Resultados y Discusión}

- Verificación de Campo

A través de una línea base obtenida de 120 encuestas aplicadas en los Municipios de San Marcos de Caiquin, Belén Gualcho y La Virtud se obtuvieron los siguientes resultados: 


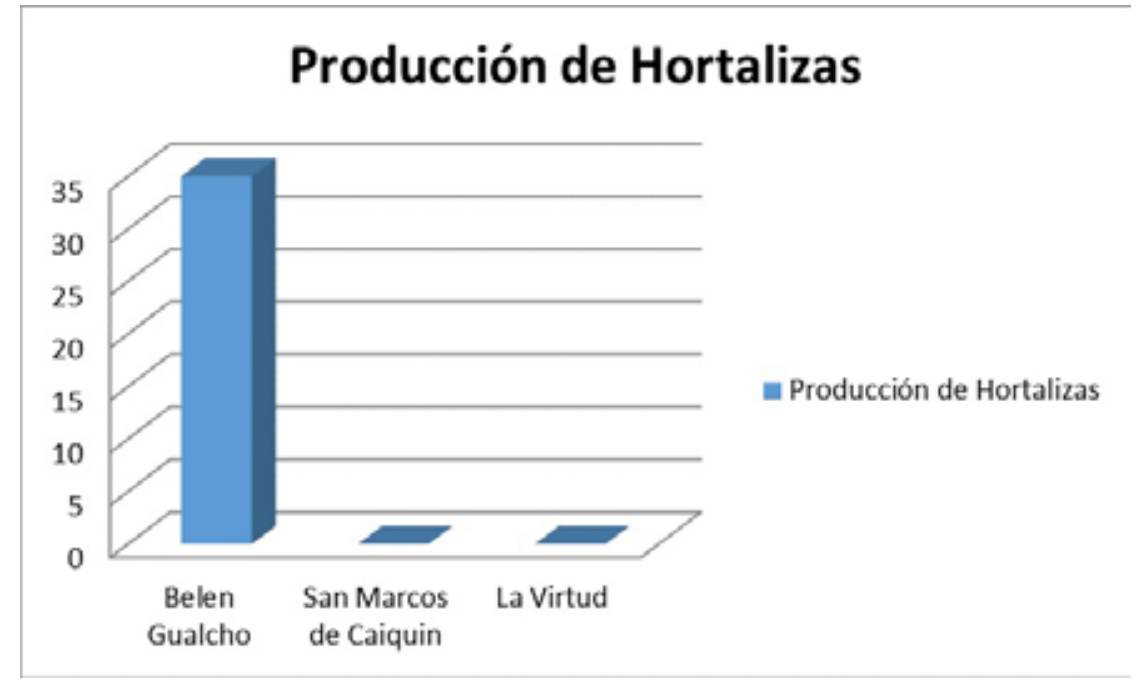

Figura 1. Se observa que el mayor productor de hortalizas a nivel de Municipios es Belén Gualcho, es así que San Marcos de Caiquin y La Virtud no producen hortalizas como tomates, pepinos, lechuga etc.

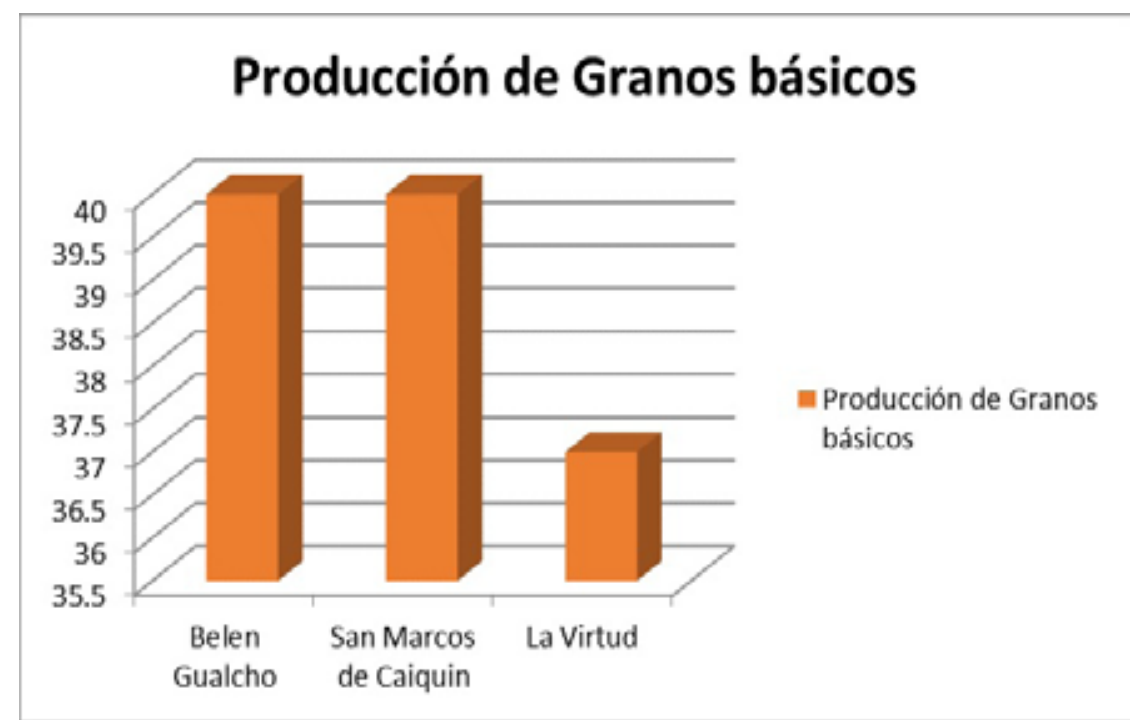

Figura 2. Podemos Observar que la mayoría o el total de productores de los Municipios de estudio producen granos básicos (maíz, frijol y maicillo). 


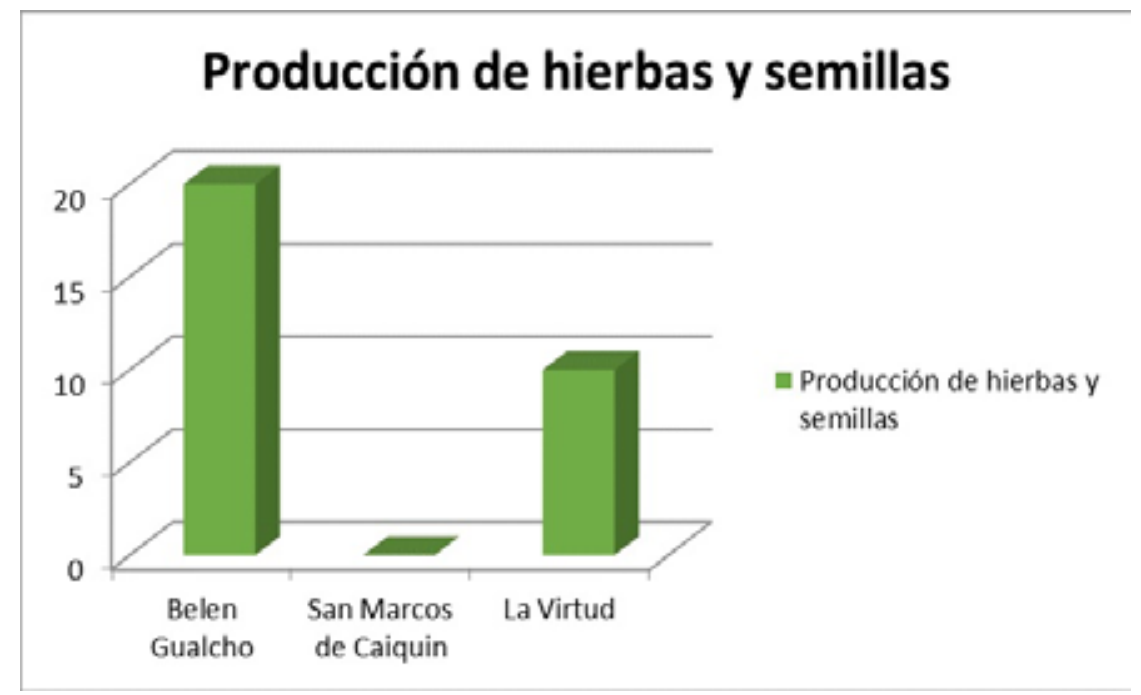

Figura 3. Belén Gualcho produce hierbas y semillas como: manzanilla, ajonjolí y chan, en cambio San Marcos de Caiquín no produce hierbas ni semillas.

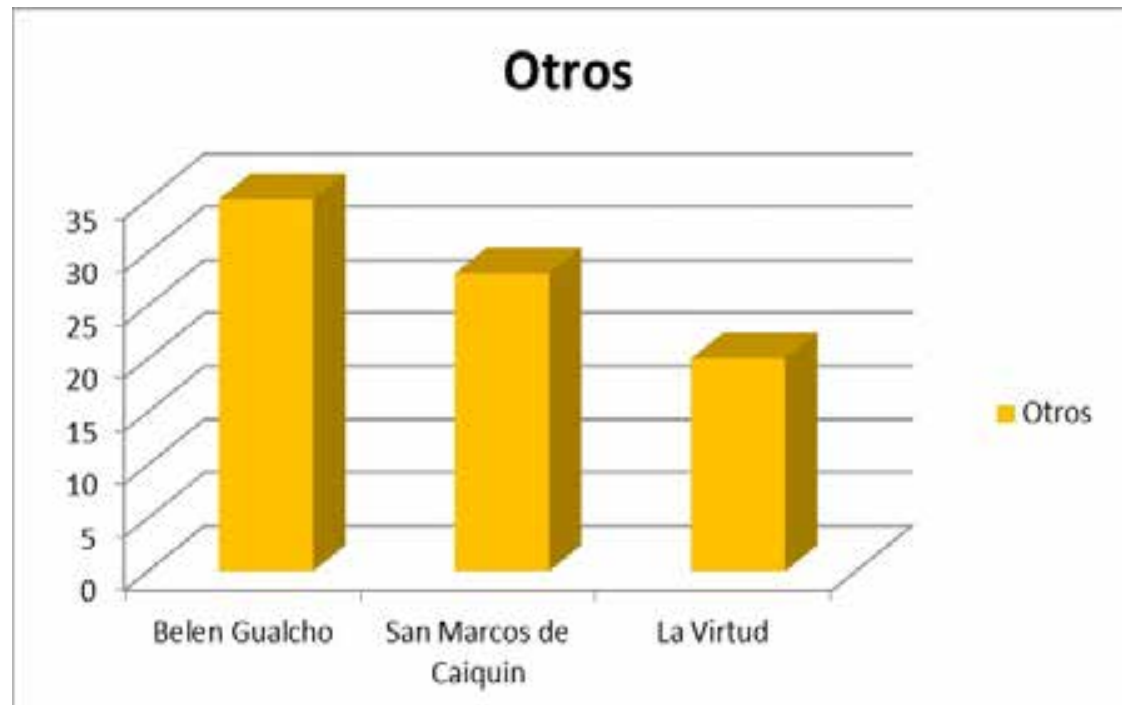

Figura 4. Todos los Municipios del estudio producen frutales (mango, naranjas, lima, limones), tubérculos (papá, yuca, malanga), arbustos. 


\section{- Identificación de Cobertura Agrícola}

Utilizando Google earth se identificaron las parcelas agrícolas del Municipio de San Marcos de Caiquin a través de observación y delimitación de polígonos, en tal sentido, logro trabajarse con el año 2014, apreciándose de forma más clara las parcelas de cultivos.

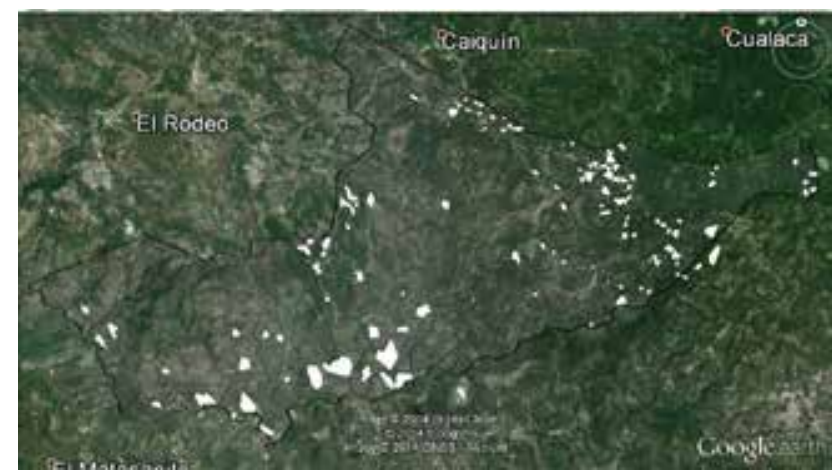

Figura 5. Muestra la imagen del 2014 donde se fueron identificando las parcelas agrícolas de San Marcos de Caiquin.

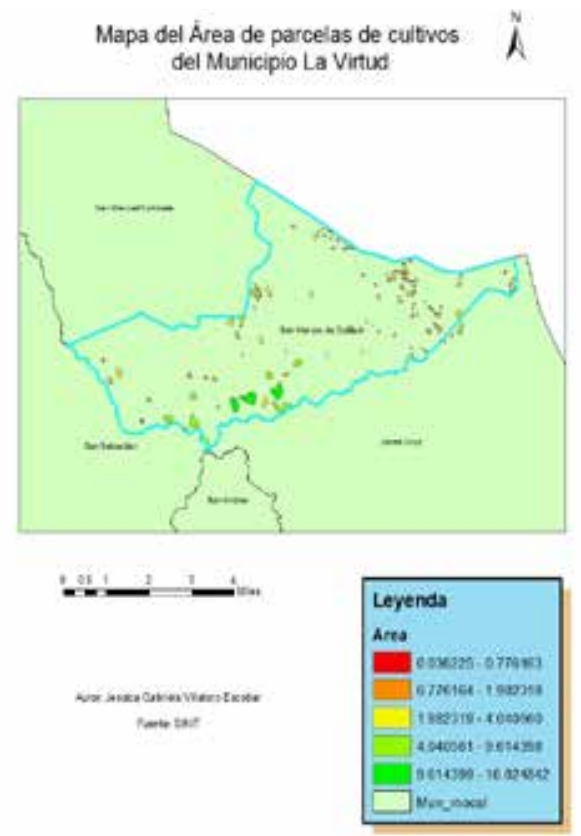

Figura 6. El color amarillo claro índica las áreas de cultivo correspondiente a un área entre 1.9 a 4 Ha y las verdes indican áreas que van de 9.6 a $16.8 \mathrm{Ha}$.. 


\section{Mapa del Área de parcelas de cultivos del Municipio Belen Gualcho \\ $\hat{\Lambda}$}
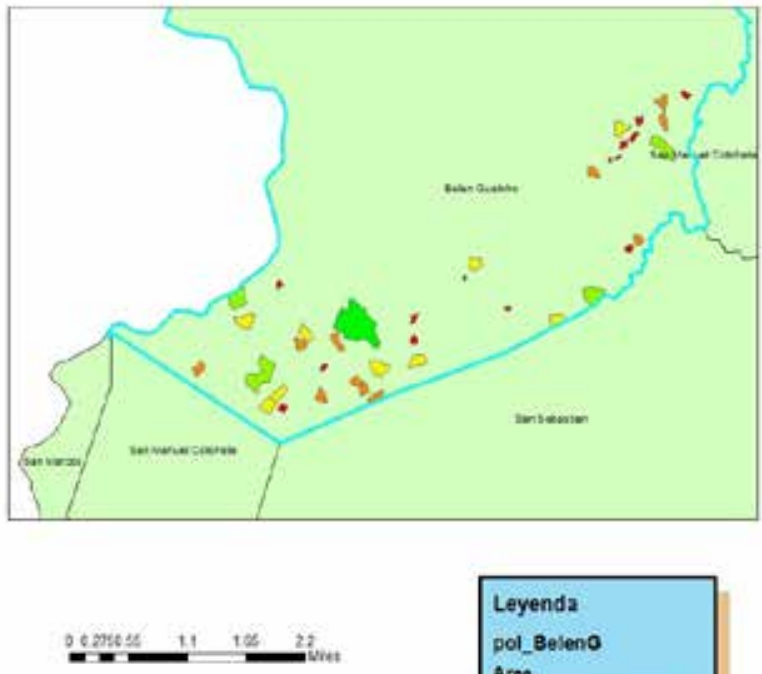

\section{Leyenda} pol_Beleno

Arse

$0000000 \cdot 1251322$ 130133.2615602

Figura 7. En el mapa de Belén Gualcho se observa que las mayores áreas de cultivo se reflejan en color verde con 4.5 a $26.03 \mathrm{Ha}$. En rojo y anaranjado las menores áreas de cultivo. 


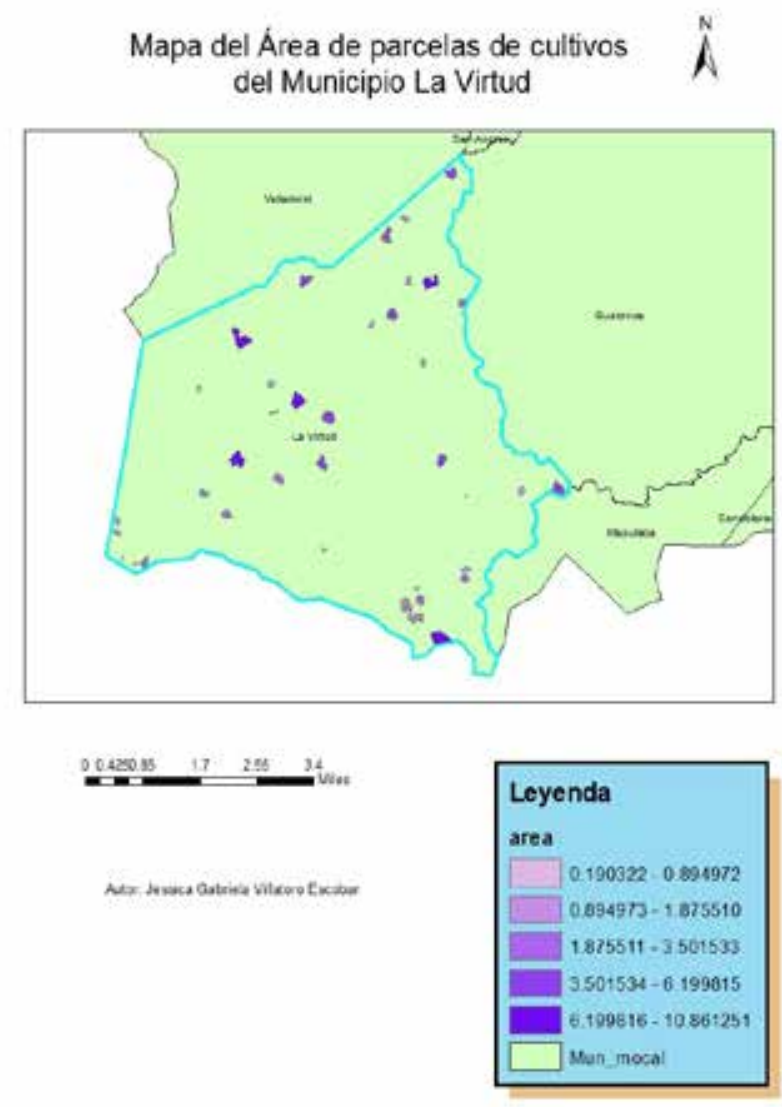

Figura 8. En el Municipio de La Virtud las mayores áreas de cultivos están representadas de 6.19 a $10.8 \mathrm{Ha}$.

\section{- Cálculo de Cobertura Agrícola}

A través del programa Arcgis 10.2 se calculó el área por parcela de cultivo identificada en el 2014, generando además un cuadro estadístico. A continuación se muestran los resultados: 


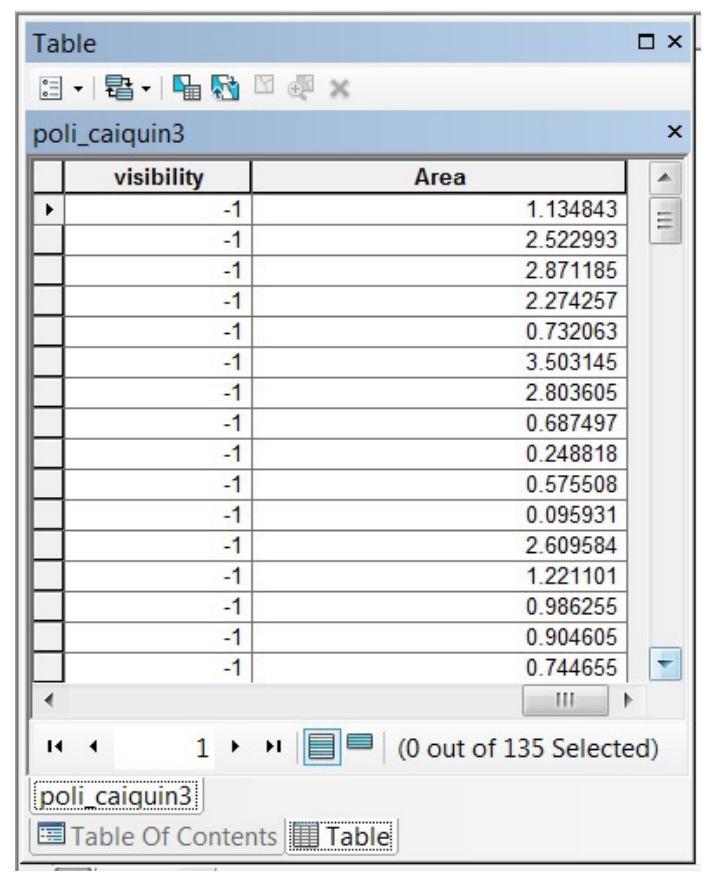

Figura 9. Aquí se muestra el cálculo del área por parcela de cultivo en el Municipio de San marcos de Caiquin, en donde la mayor área de cultivo en el Municipio corresponde a $16.8 \mathrm{Ha}$.

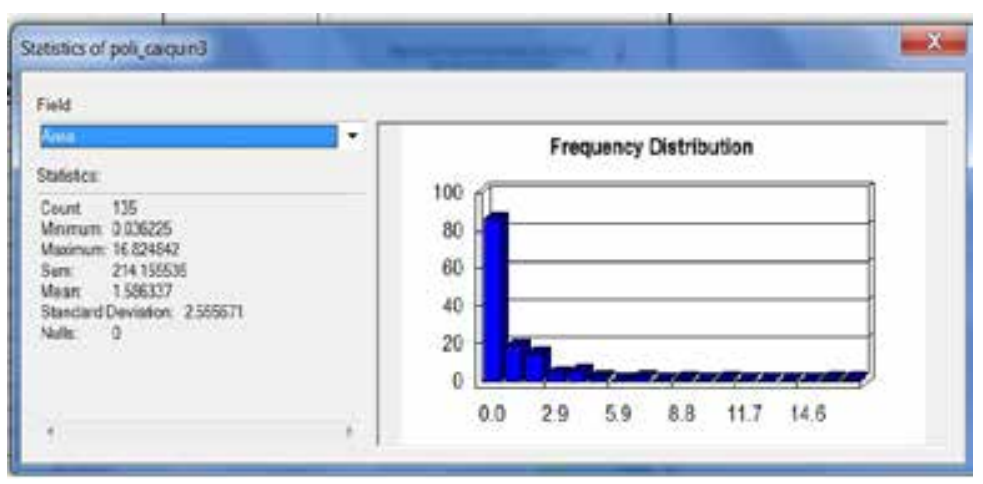

Figura 10. Distribución de frecuencia que indica que la sumatoria total de agricultura 


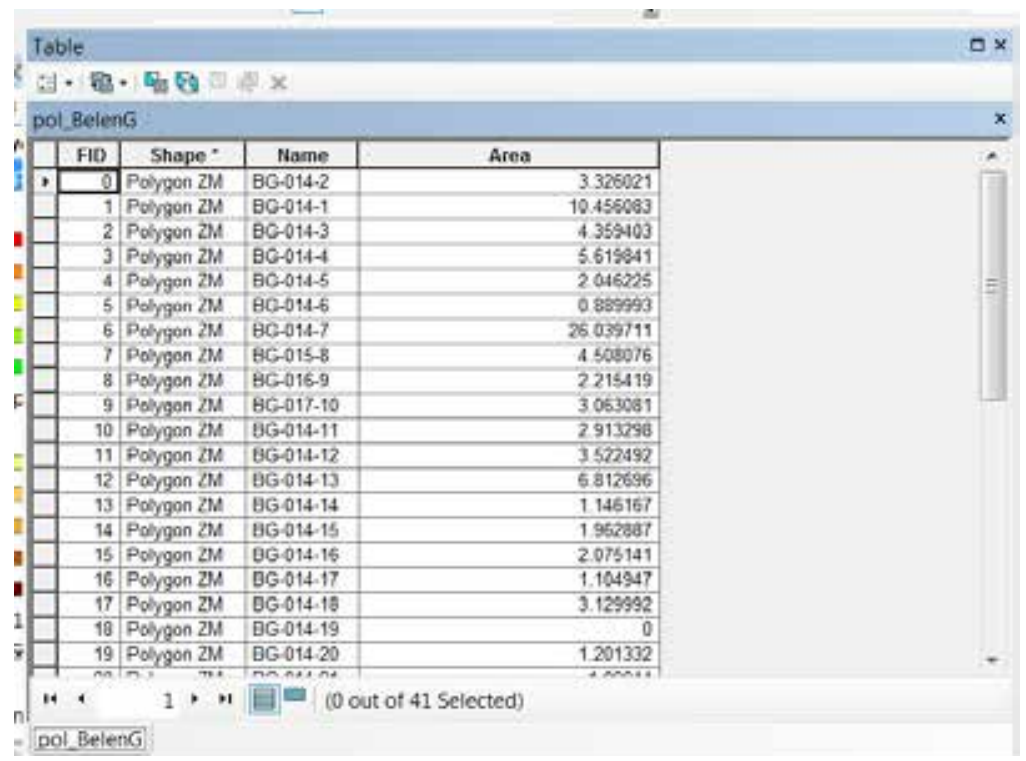

Figura 11. Aquí se muestra el cálculo del área por parcela de cultivo en el Municipio de Belén Gualcho.

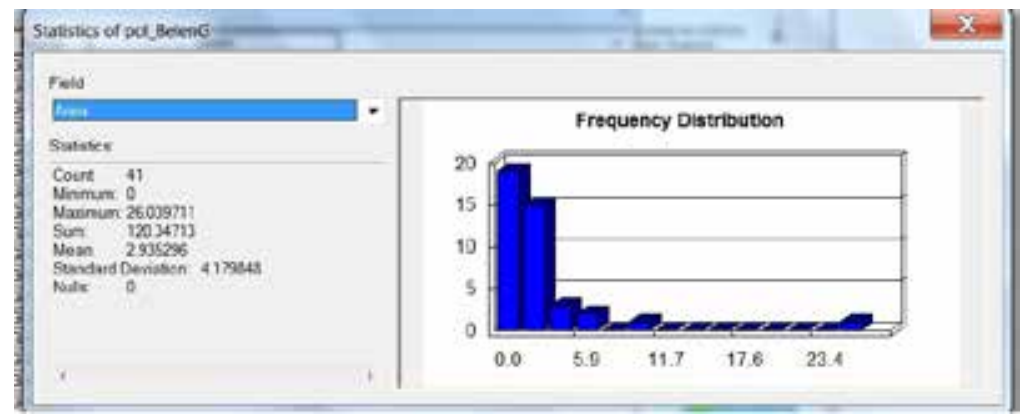

Figura 12. Distribución de frecuencia que indica que la sumatoria total de agricultura identificada correspondiente al Municipio de Belén Gualcho es de 120.34 Ha. 


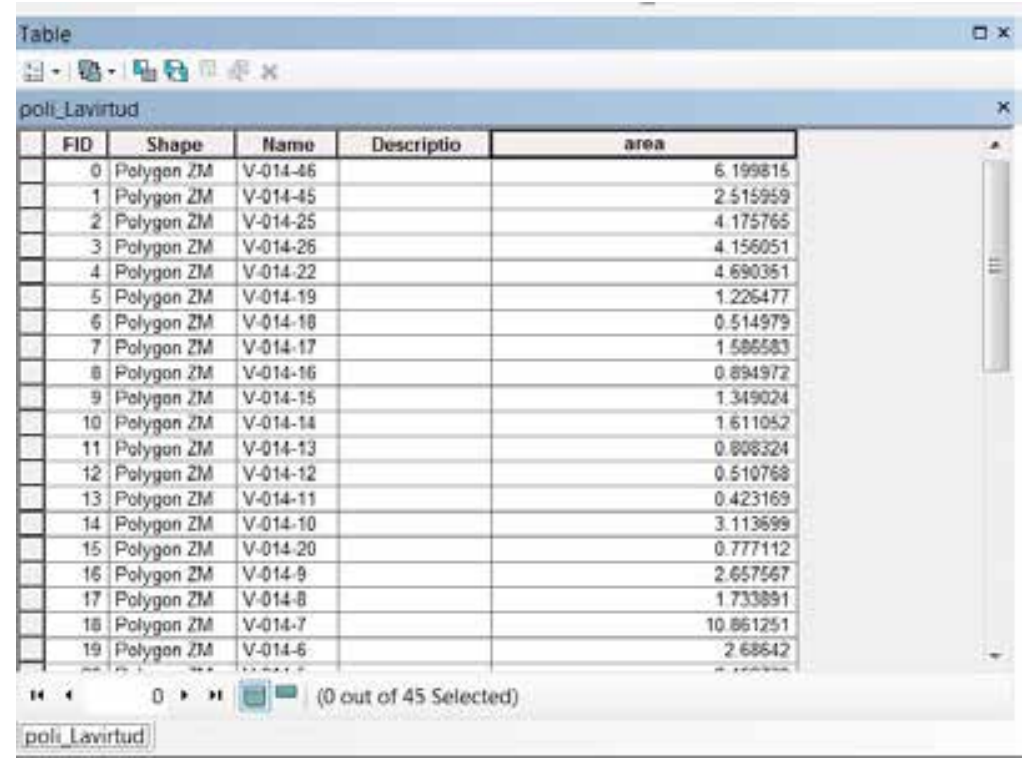

Figura 13. Aquí se muestra el cálculo del área por parcela de cultivo en el Municipio de La Virtud.

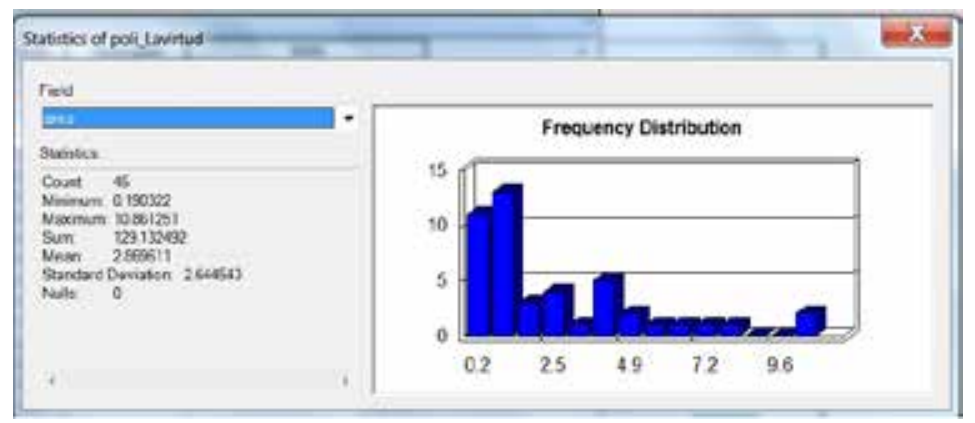

Figura 14. Distribución de frecuencia que indica que la sumatoria total de agricultura identificada correspondiente al Municipio de La Virtud es de $129.13 \mathrm{Ha} .$. 

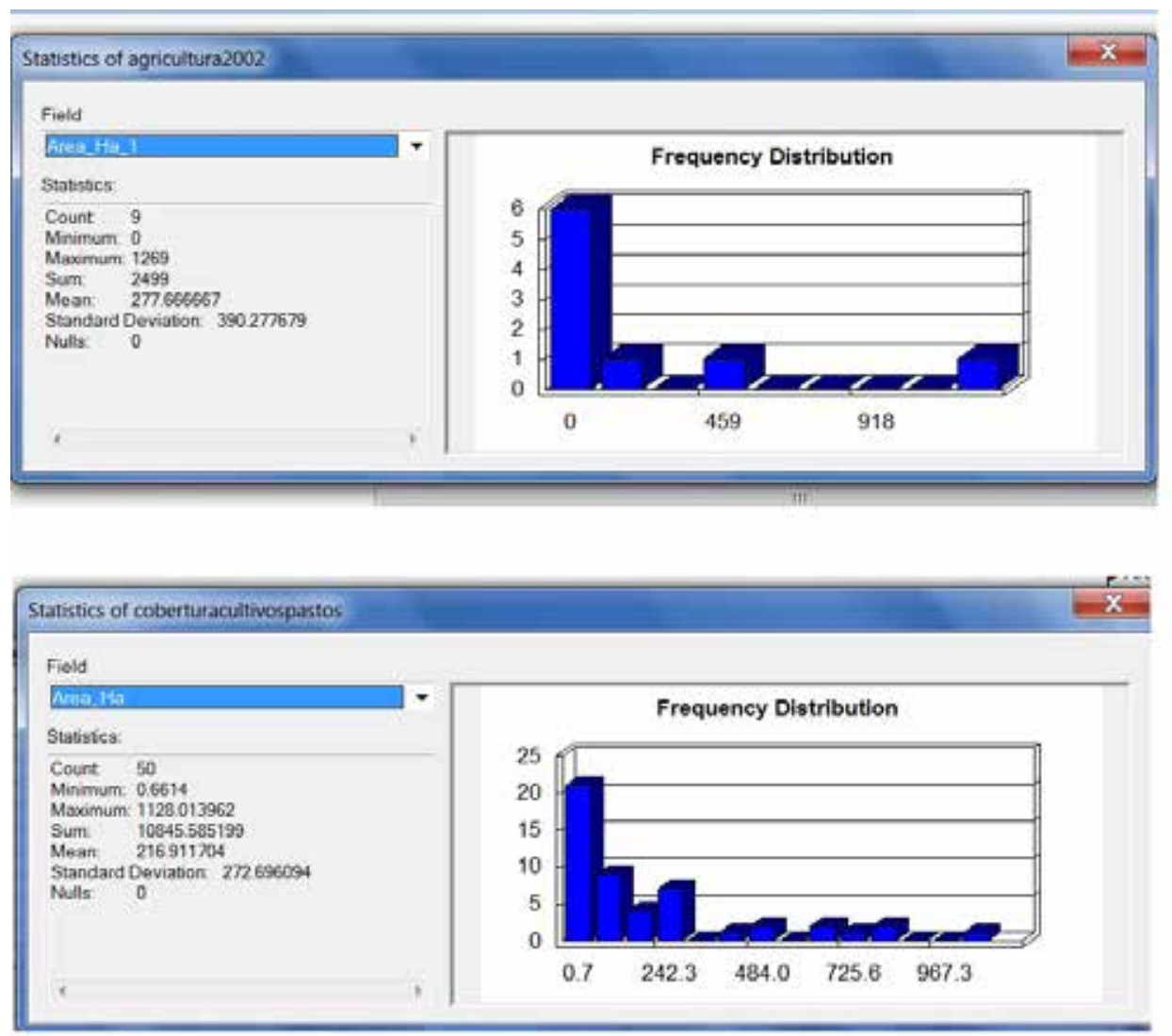

Figura 15. Cuadros estadísticos de cambio de cobertura agrícola

- Tasa de cambio de cobertura agrícola

Aplicando la fórmula:

Cambio de cobertura agrícola $=$ actual- pasada $/$ pasado $* 100$

$10845.585199-2499 * 100=333.99 \%$ 2499

El 333.99\% índica el aumento de la cobertura agrícola en un período de 12 años. 


\section{- Mapas de Cobertura Agrícola}

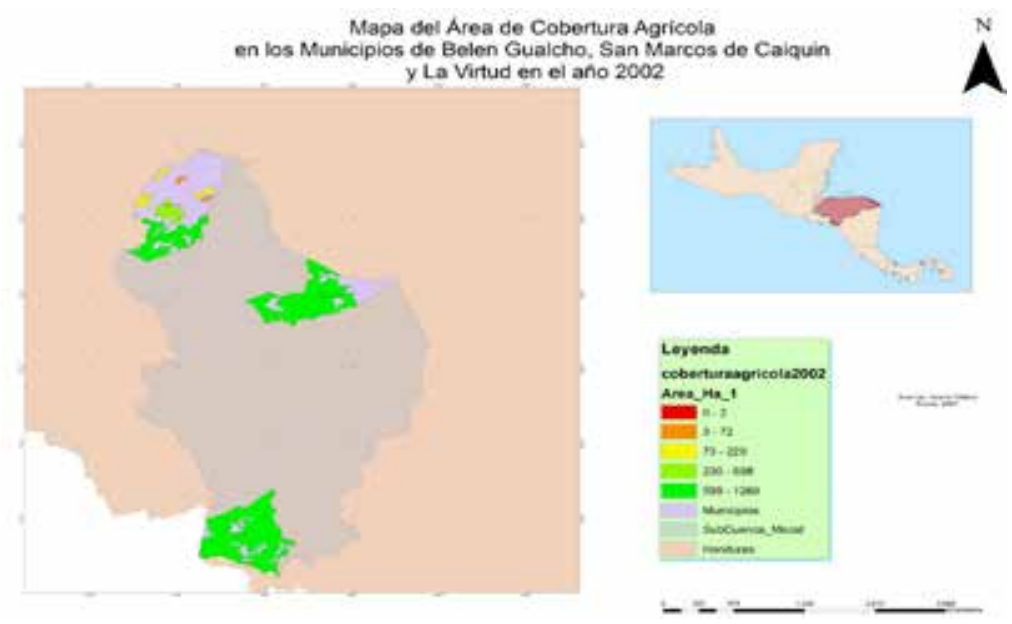

Figura 16. Mapa de cobertura agrícola del año 2002 en donde se muestra que la mayoría de área agrícola en los tres Municipios: Belén Gualcho, San Marcos de Caiquin y La Virtud oscilan con áreas entre 599-1269 Ha.

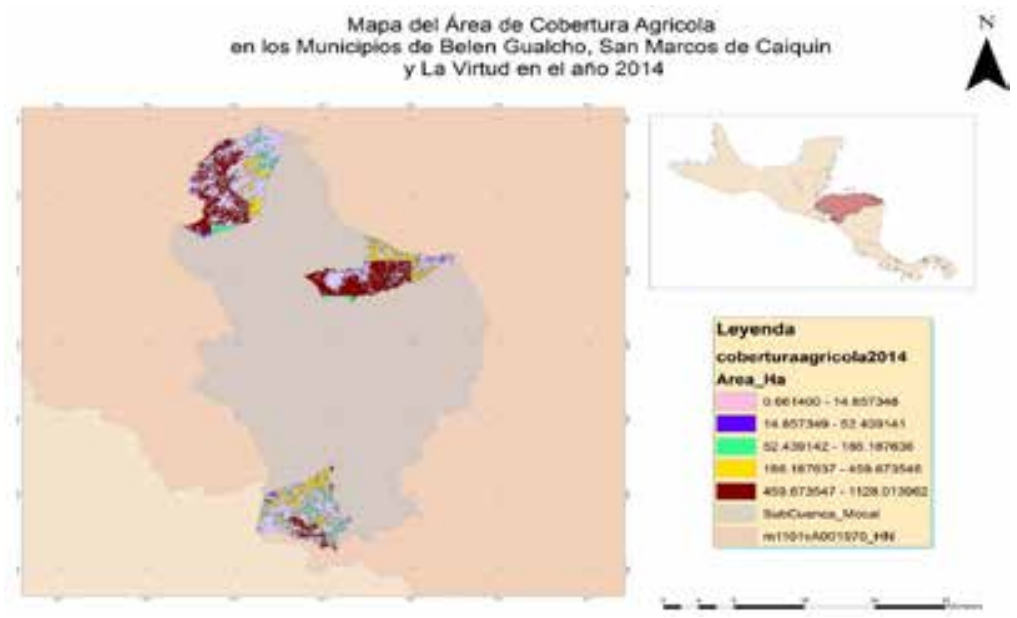

Figura 17. Mapa de cobertura agrícola del año 2014 donde los colores que más predominan en los tres Municipios (Belén Gualcho, San Marcos de Caiquin y La Virtud) son los amarillos y los marrones lo que indica que en la actualidad la cobertura agrícola entendiendo esta como pastos 0 cultivos, cuenta con áreas grandes de 186.18 a $1128.01 \mathrm{Ha}$. y La Virtud oscilan con áreas entre 599-1269 Ha. 


\section{Discusión}

En el área de estudio la mayor producción se da en granos básicos (frijoles y maíz). En San Marcos de Caiquin se reflejaron áreas que van de 9.6 a $16.8 \mathrm{Ha}$ de cobertura agrícola. En Belén Gualcho de 4.5 a 26.03 Ha y en La Virtud de 6.19 a $10.8 \mathrm{Ha}$.

En el año 2002 la cobertura agrícola total era de $2499 \mathrm{Ha}$, no obstante con el pasar del tiempo la cobertura agrícola ha ido en aumento por lo que en el 2014 se registró un total de $10845.5 \mathrm{Ha}$ de cobertura agrícola evidencia un incremento en la misma.

\section{Conclusiones}

- Al comparar el cambio de cobertura agrícola de los años 2002 al 2014 es claro identificar el aumento de cobertura agrícola en un $333.99 \%$ lo cual puede deberse al incremento de la población y por el hecho de que la agricultura es uno de los principales medios de subsistencia en el área rural, siendo los principales cultivos: Maíz y Frijoles.

- El mayor productor de Hortalizas entre los tres Municipios (Belén Gualcho, San Marcos de Caiquin y La Virtud) es Belén Gualcho que se encuentra en la parte alta de la Subcuenca del Río Mocal y que forma la línea divisoria entre Lempira y Ocotepeque.

- La cobertura agrícola está conformada en su mayoría por maíz, frijoles en toda la Subcuenca del Río Mocal, más específicamente en el área de estudio y hortalizas en la parte alta de la Subcuenca del Río Mocal. Además existen coberturas de pastos que son utilizadas para el alimento del ganado.

- Al practicar rotación de cultivos varían las coberturas, dependiendo la época se puede encontrar pasto o granos básicos en el mismo espacio de suelo pero en diferente tiempo.

\section{Recomendaciones}

- El alcance del estudio se desarrolló considerando los factores dinero y tiempo, es así que se recomienda en posteriores estudios como una segunda fase de este, realizar una validación de la interpretación en campo. 
- Siguiendo la línea de investigación se debe valorar el reemplazo de agricultura por otras coberturas como la urbanización, industria, pastos, plantaciones forestales etc.

- Para la obtención de los resultados del estudio no fue necesario realizar una clasificación, sin embargo, podría considerarse al darle otro sentido a la investigación.

\section{Bibliografía}

- Argeñal, F. J. (2010). Variabilidad Climática y Cambio Climático en Honduras. Tegucigalpa: PNUD.

- Benites, J. R., \& Alvarez Welchez, L. (1994). Ordenación Integrada de Recursos Naturales con Enfásis en Suelo, Agua y Nutrientes de Plantas. Candelaria: FAO.

- Briceño, F. (2003). Cambios de cobertura de la tierra en el valle del río Momboy. Geoenseñanza, 100.

- Castillo Altún, O. E., \& Caparó Bellido, A. (2013). Análisis multitemporal del cambio de cobertura y uso de la tierra en la MAPANCE, Honduras en el período 2000-2010. Occidente, Honduras: Zamorano.

- FAO. (marzo de 2000). Depositos de Documentos de la FAO. Obtenido de http://www.fao.org/docrep/007/ad680s/ad680s01.htm\#TopOfPage

- FAO. (16 de abril de 2005). Depositos de FAO, Departamento de Agricultura. Obtenido de Agricultura y Desarrollo Rural Sostenible: http://www.fao.org/docrep/meeting/009/j4236s/j4236s00.HTM 
- FAO. (2005). El sistema agroforestal Quesungual. Francisco Morazan: Litografia Lopez.

- FAO. (18 de 3 de 2013). Página principal de la FAO. Obtenido de http://www. fao.org/index_es.htm

- FAO, CATIE. (2000). Bibliografia comentada cambios de la cobertura forestal. Honduras: FAO.

- Fernandez, Liliana; Navarro, Edgardo; FAO. (2005). El Sistema Agroforestal Quesungual. Tegucigalpa: Litografía López.

- Hernandez, J. M., Britos, A. O., \& Barchuk, A. H. (2012). Cambios y tendencias de la cobertura/uso de la tierra en zonas áridas: Expansión de la Frontera agrícola en una cuenca del Chaco árido. Revista de la Asociación Argentina de Ecología de Paísajes, 1-21.

- IICA. (2007). Línea Base del Territorio de Belen Gualcho. Ocotepeque, Honduras: IICA.

- Indemer. (16 de octubre de 2013). Investigación de Mercados. Obtenido de http://www.colombiamercadeo.com/

- Nájera, Bojórquez, Lemus, Marceleño. (2010). Cambio de cobertura y uso del suelo en la cuenca del río Mololoa, Nayarit. Biociencias, 29.

- Santana, O. (2010). Modelagem de Espectros Temporais NDVI-MODIS. Revista Brasilera de Geografía, 47-66. 
- Tejeda, M. J. (18 de agosto de 2013). "HONDURAS SI SE PUEDE" Asegurar sus alimentos cambiando sus sistemas de producción tradicionales en laderas. Obtenido de http://mildred-tejada.rds.hn/honduras_si_puede.pdf

- Wulder, M., White, J., Gowa, S., Masek, J., Irons, J., \& Herold, M. (2008). Landsat continuity: Issues and oppotunities for land cover monitoring. Remote Sensing of Environment, 955-969. 